\title{
Pathogen versus microbiome causation in the holobiont
}

\author{
Aja Watkins ${ }^{1}$ (D) Federica Bocchi ${ }^{1}$ (I)
}

Received: 16 July 2019 / Accepted: 3 October 2019 / Published online: 24 December 2019

(c) Springer Nature B.V. 2019

\begin{abstract}
In their paper "How Causal are Microbiomes? A Comparison with the Helicobacter pylori Explanation of Ulcers," Lynch, Parke, and O'Malley successfully argue that certain causal attributions made to the microbiome have not satisfied Koch's postulates nor the interventionist framework. However, their argument involves an implicit assumption that cases such as $H$. pylori are sufficiently similar to cases involving the microbiome, such that causal attributions to both should be evaluated according to the same causal framework. Our commentary targets this assumption. First, we introduce the historical circumstances that led to the formulation of Koch's postulates in order to differentiate the case of the microbiome from that of infectious bacteria. Then, we introduce the concept of the holobiont, the idea that hosts and their symbionts should be conceptualized as a metaphysical and biological unit. Finally, using the holobiont concept, we argue that Koch's postulates and the interventionist framework are ill-equipped to handle the microbiome. We conclude by suggesting that a revision of what counts as microbiological causality within the holobiont is justified.
\end{abstract}

Keywords Microbiology $\cdot$ Microbiome $\cdot$ Holobiont $\cdot$ Biological causation

\section{Introduction}

Lynch et al. (2019) successfully argue that certain causal attributions made to the microbiome have not satisfied Koch's postulates nor the interventionist framework. However, their argument involves an implicit assumption that cases such as $H$. pylori are sufficiently similar to cases involving the microbiome, such that causal attributions to both should be evaluated according to the same causal framework.

This comment refers to the article available at https://doi.org/10.1007/s10539-019-9702-2.

Aja Watkins

ajawatki@bu.edu

1 Philosophy Department, Boston University, 745 Commonwealth Ave, Boston, MA 02215, USA 
Our commentary targets this assumption. First (Sect. 2), we introduce the historical circumstances that led to the formulation of Koch's postulates in order to differentiate the case of the microbiome from that of infectious bacteria. In Sect. 3, we introduce the concept of the holobiont, the idea that hosts and their symbionts should be conceptualized as a metaphysical and biological unit. Finally (Sect. 4), using the holobiont concept, we argue that Koch's postulates and the interventionist framework are ill-equipped to handle the microbiome.

\section{History of causal attribution in microbiology}

The postulates for attributing the cause of a disease to microbes, formulated by German physician Robert Koch around 1875, include an epistemic requirement (postulates 1 and 2) and an experimental praxis (postulate 3). The latter established the method of isolation, cultivation, and inoculation (Black 2008). This method relies on the so called one-microbe-one-disease principle, the idea that one type of microorganism is causally related to exactly one illness. This principle successfully applies in cases where the microbe is a pathogen causing an infection. Indeed, Koch was able to find out that certain bacteria (including Bacillus anthracis, Mycobacterium tuberculosis, and Vibrio cholerae) are responsible for once-widespread diseases.

Due to his success, Koch's postulates gathered momentum in assessing causality within epidemiology and they still constitute the standard for determining causation within microbiology. ${ }^{1}$ Later adjustments to the postulates are easily explained. The case of $H$. Pylori revealed a weakness with Koch's postulates: the one-microbe-onedisease relation does not always hold (Lynch et al. 2019, p. 6). This inspired a more practical way of assessing causality: the successful elimination of ulcers by antibiotics is considered sufficient evidence that $H$. Pylori is responsible for the disease.

Current studies on the microbiome, although not explicitly focused on one taxon of bacteria, rely on the same causal frameworks to establish a correspondence between the microbiome itself and a condition of the host, as Lynch et al. make clear. However, it is difficult to apply a framework developed to handle pathogens to cases of symbionts. Unlike pathogens, the human microbiome always inhabits its host. Furthermore, dysbiosis, which occurs when the normal composition of the microbiome undergoes a significant modification, is unlike an infection because the cure is to "rebalance" the microbiome (Gagliardi et al. 2018), rather than remove it. Additionally, any causal chain between the microbiome and a disease is hardly demonstrable, because dysbiosis and disease can have a common cause. This makes it difficult to establish a one-to-one relation between microbiome state and disease.

Thus, in light of these considerations, the one-bacterium-one-disease framework is ill-equipped to handle the microbiome. In the following section, we will show that the microbiome's ontological and biological dependence on the individual, understood as a holobiont, makes it hard to conceive of the former as a good candidate

\footnotetext{
${ }^{1}$ For example, they are applied today in cases such as dengue fever, influenza, SARS, and West Nile fever (Talaro and Chess 2018, p. 18, 430).
} 
for the cause of a disease in the same fashion as a pathogen can be the cause of an illness.

\section{The microbiome as part of the holobiont}

Intuitively, individual organisms are the best units for biological analysis. What an individual is, metaphysically speaking, is a matter of debate. Sometimes "organism" and "individual" are considered synonymous, and sometimes "individual" is considered to be a heterogeneous community of independent organisms working jointly to perform a common function.

The concept of the holobiont broadens the individual to include the host-a single organism-plus all of its symbionts (Rohwer et al. 2002), which function as a collective whole (Gilbert et al. 2012; O’Malley 2014, p. 156). There are several candidate holobionts, many involving microbes and a host. All legume plants depend on nitrogen-fixing microorganisms called rhizobia (e.g., Dixon and Wheeler 1986). The microorganism Wolbachia exists in co-dependent relationships with several insect species (e.g., Bordenstein et al. 2009). A human host and its microbiome may be an example of a holobiont: we are "tightly knit communities that became individuals by merger" (Margulis and Sagan 2002, p. 56). Proponents of the holobiont concept think of host organisms as not quantitatively distinct from the microbial symbionts with which their lives are entangled. A holobiont is thus an individual whose components, which are different organisms, depend on one another for their existence.

In the case of the human microbiome, considerations that favor using a holobiont instead of organism concept of the individual are based on our mutualism and evolutionary history. Unlike the infectious microbes whose study led to the formulation of Koch's postulates, the human microbiome exists in a necessarily mutualistic relationship with the human host (Talaro and Chess 2018). The normal functions of the host are made possible via interaction with the symbiont (Ley et al. 2008; Lee and Mazmanian 2010). Gut bacteria co-evolved with their host (Roughgarden et al. 2018), we vertically inherit the composition of our microbiomes from our mothers during birth (Gilbert 2014), and the microbiome can prevent colonization by other organisms (Vonaesch et al. 2018). The microbiome plays an essential, even if poorly understood, functional role throughout an individual's existence. This developmental inseparability lends itself to the suggestion that, metaphysically and biologically, we are not single organisms, but holobionts.

Given the holobiont concept, a distinction emerges between the microbiome and infectious bacteria. Infectious bacteria are better conceptualized as parasitic: metaphysically independent organisms benefiting at the expense of their host. If we apply the holobiont concept to the case of humans, the microbiome, despite not containing the same genetic material as the human host, is best conceived of as part of the individual, not something acting upon it. The microbiome is more similar to an organ, like the heart or brain, than a parasitic, infectious taxon of bacteria or other external influence. As such, any case of dysbiosis cannot be conceived of as a proper instance of a cause according to Koch's framework, just like a heart is not the proper cause of a stroke. We anticipate that Lynch et al. would agree that conditions such as obesity 
and mental health are emergent properties of the holobiont. Consequently, it will not be the case that one part of the holobiont could be the proper cause of these states, unlike if those states were caused by a parasitic microorganism.

Despite microbiomes and infectious bacteria both being studied by microbiologists, they relate in different ways to the human body, and so should be analyzed differently, as will become clear in the fourth section.

\section{Causal attribution in the holobiont}

The traditional framework does not capture the relationship between the microbiome and a disease. In this section, we show how Koch's postulates and the interventionist framework become nonsensical when the human microbiome is considered to be part of the holobiont.

If we replace "the microorganism" with "the microbiome" in Lynch et al.'s version of Koch's postulates (p. 4), ${ }^{2}$ we get:

1. The microbiome is detected in all disease cases;

2. The microbiome does not occur in other diseases or non-pathogenically;

3. After being fully isolated and repeatedly grown in pure culture, the microbiome can induce the disease by being introduced into a healthy animal host.

After this replacement, Koch's first postulate will turn out to be trivially true: the microbiome, in the case of humans, will be detected in all disease cases because it will be detected in all cases, period. Koch's second postulate will be trivially false for the same reason: the microbiome never does not occur.

Koch's third postulate, which posits that the allegedly causal microorganism can be "fully isolated" (i.e., ontologically separated) from the host, will be impracticable in the case of the microbiome (Arnold et al. 2016). The microbiome as such, namely as an ontologically-dependent group of organisms, cannot be isolated from its host; even if particular taxa or functional groups of microbes can be cultured in isolation from the host, these resulting collections of microbes cease to be a microbiome once they are isolated. Say taxa X, Y, and Z constitute the microbiome of A. Even if X, Y, and Z could be cultured separately from A, they would not thereby be a microbiome of A. The microbes' ontological status as a microbiome is dependent on their symbiosis with A.

Koch's postulates turn out to be weak in light of more recent developments in microbiology which provide a different conceptualization of what an individual is. In addition to Koch's postulates, Lynch et al. analyze causal claims according to the interventionist framework (e.g., Woodward 2010). In the interventionist framework, the purported relationship between a microbe and a disease state must be specific, stable, and proportionate.

\footnotetext{
${ }^{2}$ Koch's postulates have been formulated other ways (e.g., Black 2008, p. 13; Talaro and Chess 2018, p. 430).
} 
Claims of specificity either involve showing that the relationship between the purported cause and effect is fine-grained or that it is one-to-one. Attributing either a fine-grained or one-to-one causal relation relies on one's ability to match a variation in the cause with a corresponding variation in the effect (Lynch et al. 2019, p. 6). Here, the problem is that the microbiome - the purported cause-is extremely variable, but does not always produce variable effects. Attributing specific causation to the microbiome would involve defining a "typical" microbiome against which one could assess variation; this, though, is impossible, given that the microbiome is constantly changing in constitution, even if not in function (Odamaki et al. 2016).

Stability of a causal relationship, which involves maintenance over a range of background conditions (Lynch et al. 2019, p. 6), assumes that these background conditions don't necessarily have an effect. However, the gut microbiome and the various conditions associated with it are extremely sensitive to age (Odamaki et al. 2016), diet (Hildebrandt et al. 2009), and geography (Hooi et al. 2017), and causal claims are seldom stable across populations or species (Lynch et al., p. 8, 12). A disease state can be associated with constitutively different microbiomes in different individuals. Given the inherent instability of the microbiome itself, it is unlikely to be implicated in a stable causal relationship.

Proportionality involves selecting appropriate causes and effects, including ones that are neither too broad nor too narrow (Lynch et al. 2019, p. 6). In what sense would the whole microbiome not be too broad? If the microbiome and the various taxa within it are involved in numerous symbiotic relationships, including with the host, then it will be arbitrary to select which part of the causal chain relates to a particular effect. Causation with respect to the microbiome more closely resembles a net rather than a unidirectional chain.

Lynch et al. agree that the interventionist framework is not equipped to handle whole-microbiome causal claims:

The multi-causal nature of these traits makes it likely that there are many interactions between numerous components of the microbiome, the host, and their environments. It is for this reason that one-to-one specificity is not possible for whole microbiome explanations, and it also suggests that the causal influence of the microbiome as a whole would be extremely unstable, and difficult to isolate experimentally (p. 20).

Lynch et al. suggest that we should instead "focus causal explanations on specific microbiome components" (p. 20). We argue, however, that such specific components are not, metaphysically, the microbiome. The bacteria that constitute the microbiome may independently cause a condition in the host, but the microbiome itself, as a part of the human holobiont, cannot.

\section{Conclusion}

Lynch et al. acknowledge (p. 2) that a "microbiome revolution" is revising what we know about humans and the biological world (e.g., Blaser 2014). Applying the holobiont concept to the case of human hosts, we have argued that causal claims about 
the human microbiome are significantly dissimilar to causal claims about infectious microorganisms. A framework developed in the context of parasitism is not likely to apply seamlessly in the context of mutualism, especially not when that mutualism occurs within the holobiont. Therefore, we suggest that Koch's postulates should be replaced with ones also applicable to holobionts.

Acknowledgements Our paper greatly benefited from discussion with Professors Alisa Bokulich and Karen Warkentin. We also received helpful feedback from students in Professor Warkentin's spring 2019 Phenotypic Plasticity class.

\section{References}

Arnold JW, Roach J, Azcarate-Peril MA (2016) Emerging technologies for gut microbiome research. Trends Microbiol 24(11):887-901

Black JG (2008) Microbiology: principles and explorations, 7th edn. Wiley, Hoboken

Blaser MJ (2014) The microbiome revolution. J Clin Invest 124:4162-4165

Bordenstein SR, Paraskevopoulos C, Dunning Hotopp JC et al (2009) Parasitism and mutualism in Wolbachia: what the phylogenomic trees can and cannot say. Mol Biol Evol 26(1):231-241

Dixon ROD, Wheeler CT (1986) Nitrogen fixation in plants. Blackie \& Son, Ltd., Glasgow

Gagliardi A, Totino V, Cacciotti F et al (2018) Rebuilding the gut microbiota ecosystem. Int J Environ Res Public Health 15(8):1679

Gilbert SF (2014) A holobiont birth narrative: the epigenetic transmission of the human microbiome. Front Genet 5:282

Gilbert SF, Sapp J, Tauber AI (2012) A symbiotic view of life: we have never been individuals. Q Rev Biol 87:325-341

Hildebrandt MA et al (2009) High-fat diet determines the composition of the murine gut microbiome independently of obesity. Gastroenterology 137:1716-1724

Hooi JKY et al (2017) Global prevalence of Helicobacter pylori infection: systematic review and metaanalysis. Gastroenterology 153f:420-429

Lee YK, Mazmanian SK (2010) Has the microbiota played a critical role in the evolution of the adaptive immune system? Science 330(6012):1768-1773

Ley RE, Lozupone CA, Hamady M et al (2008) Worlds within worlds: evolution of the vertebrate gut microbiota. Nat Rev Microbiol 6:776-788

Lynch KE, Parke EC, O'Malley MA (2019) How causal are microbiomes? A comparison with the Helicobacter pylori explanation of ulcers. Biol Philos. https://doi.org/10.1007/s10539-019-9702-2

Margulis L, Sagan D (2002) Acquring genomes: a theory of the origin of species. Basic Books, New York

Odamaki T, Kato K, Sugahara H et al (2016) Age-related changes in gut microbiota composition from newborn to centenarian: a cross-sectional study. BMC Microbiol 16:90

O’Malley M (2014) Philosophy of microbiology. Cambridge University Press, Cambridge

Rohwer F, Seguritan V, Azam F, Knowlton N (2002) Diversity and distribution of coral-associated bacteria. Marine Ecol Prog Ser 243:1-10

Roughgarden J, Gilbert SF, Rosenberg E, Zilber-Rosenberg I, Lloyd E et al (2018) Holobionts as units of selection and a model of their population dynamics and evolution. Biol Theory 13:44-65

Talaro KP, Chess B (2018) Foundation in microbiology, 10th edn. Mc Graw Hill Education, New York

Vonaesch P et al (2018) Pathogens, microbiome and the host: emergence of the ecological Koch's postulates. FEMS Microbiol Rev 42:273-292

Woodward J (2010) Causation in biology: stability, specificity, and the choice of levels of explanation. Biol Philos 25:287-318

Publisher's Note Springer Nature remains neutral with regard to jurisdictional claims in published maps and institutional affiliations. 